\title{
Comportement hydrique du frêne (Fraxinus excelsior $L$ ) dans une formation montagnarde mésoxérophile
}

\author{
G Carlier, JP Peltier *, L Gielly \\ Université Joseph Fourier, laboratoire de biologie alpine, \\ BP 53 X, 38041 Grenoble cedex, France
}

(Reçu le 19 novembre 1991; accepté le 20 janvier 1992)

\begin{abstract}
Résumé - L'article décrit les variations diurnes et saisonnières du potentiel hydrique foliaire, de la conductance stomatique et de la transpiration des feuilles d'un frêne dans une station montagnarde mésoxérophile située dans la zone intermédiaire des Alpes nord-occidentales. Le potentiel hydrique de base est très proche du potentiel hydrique du sol à $30 \mathrm{~cm}$ de profondeur et en corrélation étroite avec lui. En cas de sécheresse estivale sévère, le potentiel de base peut s'abaisser à $-4,8 \mathrm{MPa}$ et le potentiel minimum à $-5,5 \mathrm{MPa}$ sans dommage pour les feuilles. En conditions favorables à l'alimentation en eau, la conductance stomatique maximale afteint $200 \mathrm{mmol}\left[\mathrm{H}_{2} \mathrm{O}\right] \mathrm{m}^{-2} \mathrm{~s}^{-1}$, valeur bien inférieure à celle des frênes des forêts alluviales de plaine. La conductance maximale ne se maintient que brièvement car une fermeture stomatique partielle a lieu au plus tard à midi (heure solaire) et éventuellement dès $7 \mathrm{~h}$. En outre, la conductance maximale diminue quand le sol s'assèche. En conséquence, le frêne de montagne consomme moins d'eau que le frêne de forêt alluviale. Cependant, sa survie au cours des périodes de sécheresse est attribuée à la stratégie de tolérance plus qu'à celle d'évitement.
\end{abstract}

Fraxinus excelsior $\mathrm{L}=$ frêne $/$ stomate $/$ potentiel hydrique $/$ sécheresse

Summary - Water relations of ash (Fraxinus excelsior $L$ ) in a mesoxerophilic mountain stand. This paper reports on the diurnal and seasonal variations in water potential, stomatal conductance, and transpiration of the leaves of an ash tree in a mesoxerophilic mountain stand situated in the intermediate zone of the north-western Alps. The pre-dawn water potential is close to the soil water potential at $30 \mathrm{~cm}$ depth and is closely correlated with the latter. In the case of pronounced summer drought the pre-dawn water potential and minimum water potential of leaves may fall as low as $-4.8 \mathrm{MPa}$ and $-5.5 \mathrm{MPa}$, respectively. Under good water supply conditions the maximum stomatal conductance may reach $200 \mathrm{mmol}\left(\mathrm{H}_{2} \mathrm{O}\right) \mathrm{mr}^{2} . \mathrm{s}^{-1}$, a value much lower than that of ash leaves in alluvial forests. The maximum stomatal conductance holds for only a short time, as stomata partially close at noon (solar time) at the latest, and in some cases as early as 7 am. Moreover, the maximum stomatal conductance decreases as the soil dries up. Accordingly ash water uptake is less in mountain stands than in alluvial forests. The survival of ash during drought is, however, attributed to tolerance strategy rather than to avoidance strategy.

Fraxinus excelsior $L=a s h /$ stoma / water potential / water stress

* Correspondance et tirés à part 


\section{INTRODUCTION}

La croissance et la productivité du frêne dépendent principalement de l'alimentation en eau à partir du sol (Devauchelle et Lévy, 1977). Les sols doivent être bien pourvus en eau, ni trop tassés, ni trop argileux, afin que les racines puissent s'enfoncer jusqu'à $1 \mathrm{~m}$ ou $1,50 \mathrm{~m}$ (Le Goff et Lévy, 1984). Le besoin en eau du frêne (masse d'eau consommée par unité de matière sèche produite) est plus élevé (Braun, 1977) et la franspiration est plus intense (Ladefoged, 1963) que pour d'autres arbres présents dans les mêmes formations que le frêne, comme l'érable sycomore.

Dans les Alpes nord-occidentales le frêne se trouve principalement dans les forêts alluviales à nappe phréatique de profondeur modérée (profondeur moyenne : 0,6-2,4 m; Pautou, 1970). Ce frêne de forêt alluviale est sujet à une transpiration très élevée pouvant atteindre $12,4 \mathrm{mmol}$ $\mathrm{m}^{-2} \cdot \mathrm{s}^{-1}$ (Besnard et Carlier, 1990), ce qui est dû à une conductance stomatique élevée (maximum : $800 \mathrm{mmol} \mathrm{m} \mathrm{m}^{-2} . \mathrm{s}^{-1}$ ), peu sensible à la température et à l'humidité atmosphérique. Cependant, le potentiel hydrique foliaire ne s'abaisse jamais audessous de $-2 \mathrm{MPa}$, valeur assez banale pour un feuillu de région tempérée (Hinckley et al, 1978).

En plus des frênaies alluviales, il existe, aux étages collinéen et montagnard inférieur, des frênaies à noisetier (Corylus avellana L) et à l'étage montagnard supérieur, des frênaies à bouleau (Betula pendula Roth) souvent installées sur des éboulis à sol peu épais et donc épisodiquemer $t$ soumises à la sécheresse estivale (Pautou et al, 1991). Le présent travail se rapporte à un frêne d'une telle formation montagnarde mésoxérophile. L'objectif est d'obtenir des informations quantitatives sur les potentiels hydriques atteints dans le sol et dans les feuilles, sur la transpiration et la conductance stomatique, afin de les comparer aux valeurs réalisées chez les frênes de forêt alluviale et de rechercher les modalités de l'adaptation du frêne montagnard à son biotope.

\section{MÉTHODES D'ÉTUDE}

\section{Site d'étude}

Le site choisi se trouve sur la commune d'Huez (Isère) à $1350 \mathrm{~m}$ d'altitude. Les coordonnées géographiques sont les suivantes : $45^{\circ} 4^{\prime} 34^{\prime \prime} \mathrm{N}$, $6^{\circ} 3^{\prime 2} 21 " \mathrm{E}$. Du point de vue phytogéographique et bioclimatique, le site appartient à la zone «intermédiaire" des Alpes nord-occidentales, définie par un angle de Gams compris entre $40^{\circ}$ et $50^{\circ}$ (Ozenda, 1985). La formation végétale est une prairie de fauche (abandonnée) à Arrhenatherum elatius $(\mathrm{L}) \mathrm{J}$ et $\mathrm{C}$ Presl et Trisetum flavescens (L) PB largement colonisée par Agropyrum campestre $\mathrm{Godr}$ et $\mathrm{Gr}$ et bocagée de frênes (Fraxinus excelsior $L$ ) et de quelques chênes sessiles (Quercus petræa [Mattuschka] Lieblein). Les frênes sont traités en taillis; les cépées sont distantes de $10-15 \mathrm{~m}$; les brins les plus forts ont 25-60 ans et 8-12 $\mathrm{m}$ de haut. La pente, orientée vers le SE, est d'environ $10 \%$.

Le sol est un limon sableux remplissant les interstices d'un éboulis de gros blocs schisteux, qui n'ont pas permis de creuser à plus de 60 $\mathrm{cm}$; l'enracinement observé ne dépasse pas 40 $\mathrm{cm}$ de profondeur.

\section{Potentiel hydrique du sol}

Le potentiel hydrique du sol a été mesuré, selon la méthode du point de rosée, au moyen de sondes hygrométriques Wescor PCT 55 placées horizontalement (Bruckler, 1984) aux profondeurs de $5,15,30,45$ et $60 \mathrm{~cm}$ et reliées à un microvoltmètre Wescor HR-33 T. Les mesures étaient faites avant le lever du soleil. Lorsqu'elles ont été répétées au cours d'une même journée, il n'a pas été constaté de variation, sauf à $5 \mathrm{~cm}$ où l'échauffement diurne rend les mesures invalides. 


\section{Conductance stomatique et potentiel hydrique foliaire}

La conductance stomatique des folioles a été déterminée à l'aide d'un poromètre Li-Cor 1600 , uniquement sur la face adaxiale (Besnard et Carlier, 1990). La résistance, affichée en s.cm-1, est convertie en conductance $g_{s}\left(\mathrm{mmol} \mathrm{m}^{-2} \mathrm{~s}^{-1}\right)$ suivant Körner et Cochrane (1985). Immédiatement après la mesure porométrique la foliole était détachée et son potentiel hydrique $\psi_{f}$ mesuré à l'aide d'une bombe de pression (Scholander et al, 1965). Toutes les heures 5 folioles appartenant à des feuilles différentes étaient traitées successivement. De telles séries horaires étaient répétées 11 à 14 fois au cours de la journée. Toutes les folioles utilisées étaient adultes et ont été choisies dans le quadrant sud d'une même cépée, entre 2,5 et $3,5 \mathrm{~m}$ de hauteur sur des brins de $15 \mathrm{~cm}$ de diamètre au moins et d'environ $9 \mathrm{~m}$ de haut.

\section{Conditions microclimatiques}

Au début et à la fin de chaque série horaire, la température et l'humidité de l'air étaient déterminées à proximité du feuillage (mais à l'abri du soleil) à l'aide d'un thermo-hygromètre Coreci, régulièrement recalé suivant la notice du constructeur. L'éclairement (ou irradiance, en $\mu \mathrm{mol}$ [photons] $\mathrm{m}^{-2} . \mathrm{s}^{-1}$ ) reçu par chaque foliole, perpendiculairement au limbe, était mesuré à l'aide d'un capteur Li-Cor 190-SB installé sur le poromètre.

Les précipitations indiquées sont celles du poste météorologique de Bourg d'Oisans, distant de $4 \mathrm{~km}$.

\section{Calcul de la transpiration}

La transpiration mesurée par le poromètre est considérée comme différente de la transpiration réelle de la foliole à l'air libre, car l'atmosphère de la cuvette est plus chaude et plus sèche que l'air ambiant. La transpiration $\left(E_{c a l}\right.$, en mmol.m-2. $\mathrm{s}^{-1}$ ) a donc été calculée en tenant comple de la pression de vapeur d'eau dans l'air, de la pression de vapeur saturante à la température de la foliole et de la résistance stomatique donnée par le poromètre; la résistance de la couche limite a été prise égale à celle existant dans le poromètre, soit $0,2 \mathrm{~s} . \mathrm{cm}^{-1}$.

Les valeurs retenues pour la conductance stomatique, la transpiration et le potentiel hydrique sont les moyennes de chaque série horaire.

\section{Relations entre RWC et potentiels}

Les relations entre la teneur en eau relative (RWC \%, Turner et Kramer, 1980) et les potentiels hydrique $\psi_{f}$ et osmotique $\psi_{s}$ ont été déterminées, à des dates choisies, sur des lots de folioles. Les folioles ont d'abord été saturées $\left(\psi_{f}=\right.$ $0, \mathrm{RWC}=100 \%$ ) par séjour de $48 \mathrm{~h}$ en atmosphère saturée, à $5{ }^{\circ} \mathrm{C}$, la base du limbe étant immergée dans de l'eau distillée. Plusieurs folioles saturées ont été tuées par immersion dans l'azote liquide et leur potentiel osmotique à l'état saturé $\psi_{s o}$ a été déterminé dans des chambres en inox munies de sondes psychrométiques Wescor PST 55 reliées à un microvoltmètre Wescor PR 55 (une foliole par chambre). Les folioles restantes ont été amenées à différents niveaux de RWC et on a mesuré leur potentiel hydrique $\psi_{f}$ puis, après passage dans l'azole liquide, leur potentiel osmotique $\psi_{s}$. Dans une série décroissante de RWC, le premier RWC pour lequel $\psi_{s}=\psi_{f}$ indique, par défaut, le début de perte de turgescence; le potentiel correspondant est $\psi_{s l}$.

\section{Tests statistiques}

Les corrélations ont été éprouvées par le test de Pearson ou, lorsque celui-ci est illicite, par le test de Kendall (Sokal et Rohlf, 1981).

\section{RÉSULTATS}

\section{Les conditions hydriques du milieu et le potentiel foliaire de base (fig 1)}

Les mesures ont commencé le 16 août 1988 à la suite d'une période de 6 se- 


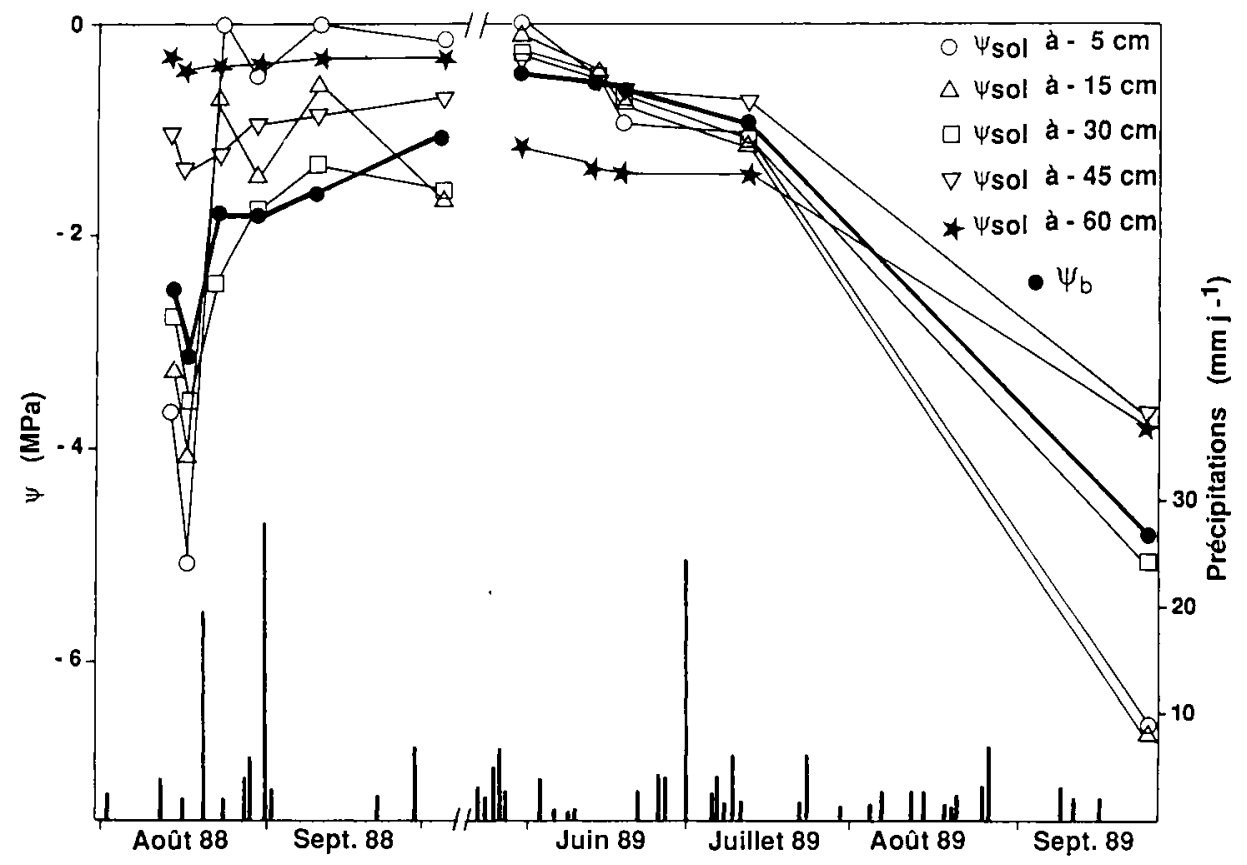

Fig 1. Marche saisonnière du potentiel hydrique du sol $\psi_{\text {sol }}$ à 5 profondeurs et du potentiel hydrique de base $\psi_{b}$ des feuilles de frêne (en haut). Précipitations (en bas).

maines caractérisée par des précipitations réduites; le sol était $\sec \left(\psi_{\text {sol }}=-2,73 \mathrm{MPa}\right.$ à $30 \mathrm{~cm}$ de profondeur le 16 août 1988) et le gradient des potentiels hydriques croissant vers la profondeur. La pluie du 20 août 1988 a fait remonter les potentiels, instantanément en surface et plus progressivement en profondeur.

La campagne de mesures de 1989 a commencé fin mai, sur sol bien humide en surface avec un gradient de potentiel légèrement décroissant vers la profondeur. L'été n'a comporté que des précipitations minimes de juillet à septembre, l'assèchement du sol à partir de la surface entraînant l'inversion du gradient. En fin de saison, le sol était extrêmement $\sec \left(\psi_{\text {sol }}=-\right.$ $5,09 \mathrm{MPa}$ à $30 \mathrm{~cm}$ de profondeur le 27 septembre 1989). L'été 1990 a connu une sécheresse moins poussée $\left(\psi_{\text {sol }}=-1,95\right.$ MPa à $-30 \mathrm{~cm}$ le 7 septembre 1990).

Les mesures physiologiques ont donc pu être faites dans un intervalle très large de conditions hydriques édaphiques. Le potentiel de base des feuilles $\psi_{b}$ suit les variations de potentiel du sol. En fait il est, sauf rares exceptions, compris entre les potentiels du sol à 30 et à $45 \mathrm{~cm}$ de profondeur (fig 1). Le $\psi_{b}$ est très étroitement corrélé au $\psi_{\text {sol }}$ à $-30 \mathrm{~cm}(r=0,984 ; n=$ 11; $P<0,01)$. Les corrélations de $\psi_{b}$ avec les $\psi_{\text {sol }}$ aux autres profondeurs sont moins bonnes. II n'y a pas de corrélation entre $\psi_{b}$ et le $\psi_{\text {sol }}$ à $-60 \mathrm{~cm}$, profondeur à laquelle il n'a pas été observé de racines. 
Même quand $\psi_{b}$ était au plus bas $(-3,16$ MPa le 18 août 1988 et $-4,80 \mathrm{MPa}$ le 27 septembre 1989) les feuilles paraissaient turgescentes et ne présentaient pas de signes de dommages. Cependant quelques frênes voisins ont subi des dommages se manifestant pas le brunissement de certaines feuilles.

\section{Schémas des variations diurnes des grandeurs physiologiques}

Toutes les journées de mesures se sont déroulées par temps bien à très bien ensoleillé. La figure 2 donne 4 exemples de variations des grandeurs physiologiques, choisis pour couvrir l'ensemble des conditions hydriques rencontrées, des plus modérées $\left(\psi_{b}=-0,48 \mathrm{MPa}\right.$ le 31 mai 1989) aux plus sévères $\left(\psi_{b}=-4,8 \mathrm{MPa}\right.$ le 27 septembre 1989). Les valeurs extrêmes pour toutes les journées sont portées au tableau I.

La conductance stomatique, faible mais rarement nulle avant le lever du soleil, augmente avec l'éclairement et culmine dans la matinée (fig 2). Le maximum de conductance tend à être plus précoce en période sèche qu'en période humide; par exemple il s'est produit à $8 \mathrm{~h}$ le 18 août $1988\left(\psi_{b}=\right.$ $-3,16 \mathrm{MPa})$ et à $12 \mathrm{~h}$ le 31 mai $1989\left(\psi_{b}=\right.$ $-0,48 \mathrm{MPa}$ ). La conductance décroît ensuite de 30 à $75 \%$, en $1-2 \mathrm{~h}$, tandis que l'éclairement continue à augmenter. Elle reste alors plus ou moins constante ou décroît doucement jusqu'à la fin de la journée, où la fermeture des stomates s'accentue. Le 27 septembre $1989\left(\psi_{b}=-4,8\right.$ $\mathrm{MPa}$ ) les stomates se sont très peu (et cependant significativement) ouverts et le moment du maximum de conductance est imprécis.

Le maximum de transpiration se produit en milieu de journée. II est toujours postérieur au maximum de conductance, ce qui résulte évidemment du fait que la température et le VPD atmosphériques continuent à augmenter alors que la conductance diminue. Quant au minimum de potentiel hydrique foliaire, il est atteint en milieu de journée ou plus tard. II coïncide le plus souvent avec le maximum de transpiration $\grave{a} \pm 1 \mathrm{~h}$ près (ce qui représente le pas de temps des mesures). Cependant les 18 et

Tableau I. Valeurs extrêmes journalières des grandeurs physiologiques.

\begin{tabular}{|c|c|c|c|c|c|c|c|c|}
\hline \multirow[t]{2}{*}{ Dates } & \multirow{2}{*}{$\begin{array}{l}\psi_{b} \\
M P a\end{array}$} & \multicolumn{2}{|c|}{$g_{s \max }$} & \multicolumn{2}{|c|}{$E_{c a l}$ maximal } & \multicolumn{2}{|c|}{$\psi_{f \min }$} & \multirow{2}{*}{$\begin{array}{l}\Delta \psi \\
M P a\end{array}$} \\
\hline & & $\begin{array}{l}\text { valeur } \\
\mathrm{mmol} .\end{array}$ & $\begin{array}{l}\text { heure } \\
T^{2} \cdot s^{-1}\end{array}$ & $\begin{array}{l}\text { valeur } \\
\text { mmol.r }\end{array}$ & $\begin{array}{l}\text { heure } \\
m^{-2} \cdot s^{-1}\end{array}$ & $\begin{array}{l}\text { valeur } \\
\mathrm{MPa}\end{array}$ & heure & \\
\hline 16 août 1988 & $-2,50$ & 55,0 & 7 h 00 & 0,860 & 11 h 15 & & & \\
\hline 18 août 1988 & $-3,16$ & 79,8 & 8 h 15 & 0,620 & 9 h 15 & $-4,18$ & $16 \mathrm{~h} 10$ & 1,02 \\
\hline 24 août 1988 & $-1,77$ & 127,4 & $7 \mathrm{~h} 15$ & 0,843 & $13 \mathrm{~h} 20$ & $-2,66$ & $13 \mathrm{~h} 20$ & 0,89 \\
\hline 31 août 1988 & $-1,82$ & 70,6 & 8 h 30 & 0,978 & 11 h 30 & $-3,08$ & 14 h 20 & 1,26 \\
\hline $\begin{array}{l}12 \text { sept } 1988 \\
5 \text { oct } 1988\end{array}$ & $\begin{array}{l}-1,59 \\
-1,06\end{array}$ & 135,2 & 9 h 10 & 1,255 & $13 \mathrm{~h} 10$ & $-2,67$ & 14 h 05 & 1,08 \\
\hline 31 mai 1989 & $-0,48$ & 200,2 & 12 h 00 & 2,900 & 12 h 00 & $-1,13$ & $12 \mathrm{~h} 00$ & 0,65 \\
\hline 14 juin 1989 & $-0,51$ & 190,4 & 9 h 30 & 2,532 & 12 h 30 & $-1,48$ & $12 \mathrm{~h} 30$ & 0,97 \\
\hline 19 juin 1989 & $-0,65$ & 117,9 & 7 h 30 & 1,703 & $11 \mathrm{~h} 30$ & $-1,75$ & $11 \mathrm{~h} 30$ & 1,10 \\
\hline 13 juill 1989 & $-0,93$ & 160,9 & 8 h 30 & 2,015 & 12 h 30 & $-2,27$ & $11 \mathrm{~h} 40$ & 1,34 \\
\hline 27 sept 1989 & $-4,80$ & 6,6 & 13 h 10 & 0,127 & 13 h 10 & $-5,54$ & $14 \mathrm{~h} 10$ & 0,74 \\
\hline
\end{tabular}




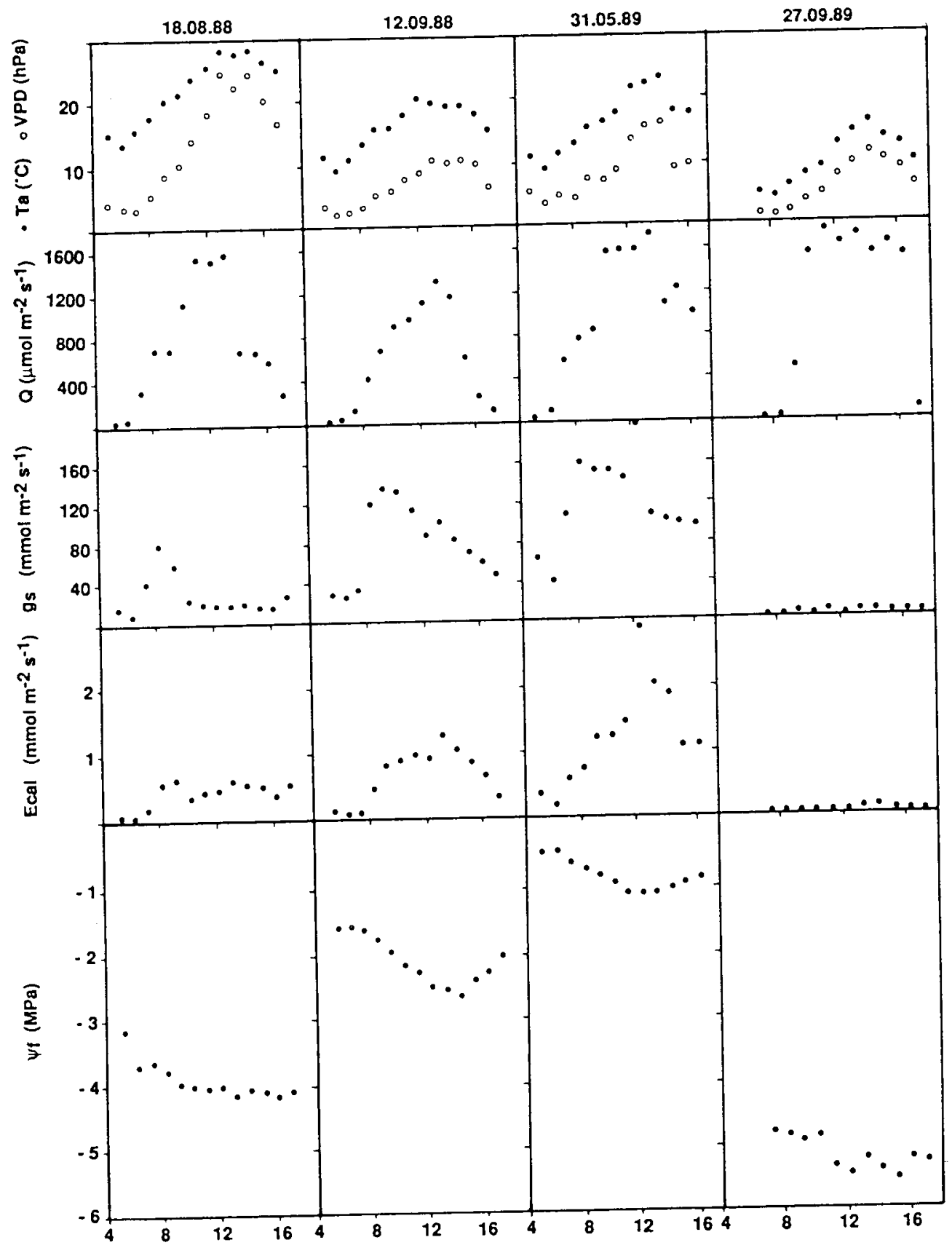


31 août 1988 , le minimum de $\psi_{f}$ est nettement plus tardif mais cela correspond à des courbes de variation de la transpiration très aplaties.

\section{Relation de la conductance avec l'éclairement reçu par les feuilles}

Si, pour chaque journée, on traçait le graphe des points représentant la conductance en fonction de l'éclairement sans tenir compte de leur ordre chronologique, ces points s'ajusteraient, avec une dispersion notable, à des courbes d'allure hyperbolique comportant un plateau quasi horizontal atteint pour des éclairements compris entre 400 et $800 \mu \mathrm{mol}$ (photons) $\mathrm{m}^{-2} \cdot \mathrm{s}^{-1}$. Mais si on relie les moyennes horaires dans l'ordre chronologique (fig 3) on obtient des boucles qui peuvent être décomposées en 5 segments comme suit :

- a) une phase d'ouverture d'une durée de 2-3 h; nous appelons "efficacité lumineuse" la pente moyenne de ce segment;

- b) un plateau correspondant approximativement à la conductance maximale et pouvant se réduire à celle-ci (par exemple le 18 août 1988 , fig 3);

- c) un décrochement constitué par la diminution de conductance sous éclairement constant ou croissant et réalisé en 1-2 h;

- d) une phase de "conductance stabilisée", sous éclairement décroissant, qui dure plusieurs h jusqu'à ce que l'éclairement retombe au voisinage de $200 \mu \mathrm{mol}$ (photons) $\mathrm{m}^{-2} \cdot \mathrm{s}^{-1}$;

- e) la fermeture finale dont le tracé se confond avec celui de l'ouverture matinale.
Cette phase n'est observée que si les mesures sont prolongées suffisamment tard.

Tout se passe comme s'il existait 2 relations distinctes de $g_{s}$ avec l'éclairement, l'une s'appliquant le matin jusqu'au décrochement $(a, b)$ et l'autre après le décrochement (d, e). Le 27 septembre 1989 les conductances sont tellement faibles que les 2 relations sont confondues. Le passage d'une relation à l'autre suggère l'intervention, directe ou indirecte, de facteurs autres que l'éclairement.

\section{Relations de la conductance avec des variables autres que l'éclairement}

L'étude des conditions microclimatiques (température, VPD) existant au moment où

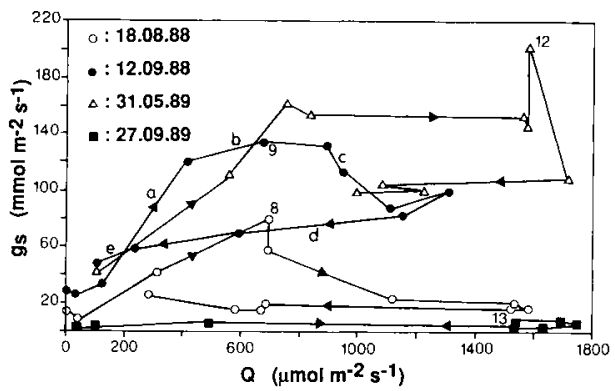

Fig 3. Quatre exemples (mêmes dates que pour la fig 2) de relation entre la conductance stomatique $g_{s}$ et l'éclairement reçu par la feuille $Q$. Chaque point représente la moyenne de 5 mesures. Les courbes se lisent dans le sens chronologique indiqué par les flèches (un point par heure). L'heure du maximum de conductance est indiquée à côté du point correspondant.

Fig 2. Quatre exemples de cinétique diurne des conditions microclimatiques (température, $T_{a}$; VPD, éclairement; $Q$ ) et des paramètres physiologiques foliaires (conductance, $g_{s} ;$ transpiration, $\mathrm{E}_{\mathrm{cal}} ;$ potentiel hydrique, $\psi_{i}$ ). Chaque point représente la moyenne de 5 mesures (sauf pour $T_{a}$ et VPD : 2 mesures). 
la conductance est maximale ou au début du "décrochement» (les 2 étant occasionnellement confondus) ne fait apparaître aucune coïncidence remarquable (résultats non présentés). En revanche plusieurs corrélations significatives, voire très significatives, ont été mises en évidence (tableau II):

- la conductance maximale $g_{\text {smax }}$ diminue très significativement avec le potentiel de base $\psi_{b}\left(\tau=0,778^{\star \star}\right)$; elle diminue aussi avec le potentiel hydrique foliaire qui lui est concomitant; mais cette relation est moins étroite que la précédente $(\tau=$ $\left.0,666^{\star}\right)$;

- la "conductance stabilisée" est très étroitement corrélée au potentiel de base $\left(\tau=0,911^{* \star}\right)$; cette relation peut n'être pas indépendante de la précédente car la conductance maximale et la conductance stabilisée sont elles-mêmes en étroite corrélation $\left(\tau=0,778^{\star *}\right)$;
- l'efficacité lumineuse décroît avec le $\psi_{b}$ $\left(\tau=0,688^{*}\right)$; cette relation est indépendante de celle qui relie $g_{\text {smax }}$ à $\psi_{b}$, car $g_{\text {smax }}$ n'est pas corrélée avec l'efficacité lumineuse (autrement dit, ce n'est pas parce que la conductance augmente vite à partir du lever du soleil qu'elle atteindra une valeur maximale élevée).

Ainsi la sécheresse entraîne une diminution de l'ensemble des valeurs de la conductance et une détérioration de la réponse des stomates à l'éclairement.

\section{Relation entre le potentiel $\psi_{f}$ et la transpiration $E_{c a l}$ (fig 4)}

Les courbes exprimant cette relation s'étagent exactement dans l'ordre des potentiels de base, ce qui montre qu'en toute circonstance le $\psi_{f}$ dépend d'abord des conditions édaphiques et ensuite seulement de la transpiration.

Tableau II. Corrélations des paramètres stomatiques et de la conductance hydraulique avec le potentiel de base.

\begin{tabular}{|c|c|c|c|c|c|}
\hline Dates & $\begin{array}{l}\psi_{b} \\
M P a\end{array}$ & $\begin{array}{l}g_{\text {smax }} \\
\text { mmol.m-m.s.s } s^{-1}\end{array}$ & $\begin{array}{l}g_{s} \text { Stabilisée } \\
\text { mmol.m-2.s.s }\end{array}$ & $\begin{array}{l}\text { Efficacité } \\
\text { lumineuse } \\
\left.\text { mmol[ } \mathrm{H}_{2} \mathrm{O}\right] \text { : } \\
\text { Imol[photons] }\end{array}$ & $\begin{array}{l}\text { Conductance } \\
\text { hydraulique } \\
\text { mmol. } m^{-2} \cdot s^{-1} \\
M^{-1}\end{array}$ \\
\hline 16 août 1988 & $-2,50$ & 55,0 & 26 & 0,119 & \\
\hline 18 août 1988 & $-3,16$ & 79,8 & 20 & 0,112 & 0,927 \\
\hline 24 août 1988 & $-1,77$ & 127,4 & 45 & 0,280 & 1,047 \\
\hline 31 août 1988 & $-1,82$ & 70,6 & 34 & 0,168 & 0,925 \\
\hline 12 sept 1988 & $-1,59$ & 135,2 & 70 & 0,288 & 1,156 \\
\hline 31 mai 1989 & $-0,48$ & 200,2 & 100 & 0,200 & 3,745 \\
\hline 14 juin 1989 & $-0,51$ & 190,4 & 110 & 0,603 & 2,597 \\
\hline 19 juin 1989 & $-0,65$ & 117,9 & 80 & 0,375 & 2,045 \\
\hline 13 juill 1989 & $-0,93$ & 160,9 & 90 & 0,940 & 1,587 \\
\hline 27 sept 1989 & $-4,80$ & 6,6 & 4 & 0,004 & 0,223 \\
\hline$\tau$ de Kendall & & $0,778^{* * 1}$ & $0,911^{* *}$ & $0,688^{*}$ & $0,943^{* * 2}$ \\
\hline
\end{tabular}

$1{ }^{* *} P \leq 0,01 ; *: 0,01<P \leq 0,05 ;{ }^{2}$ Corrélation entre la conductance hydraulique et $g_{s \max } t=0,833^{* *}$. 


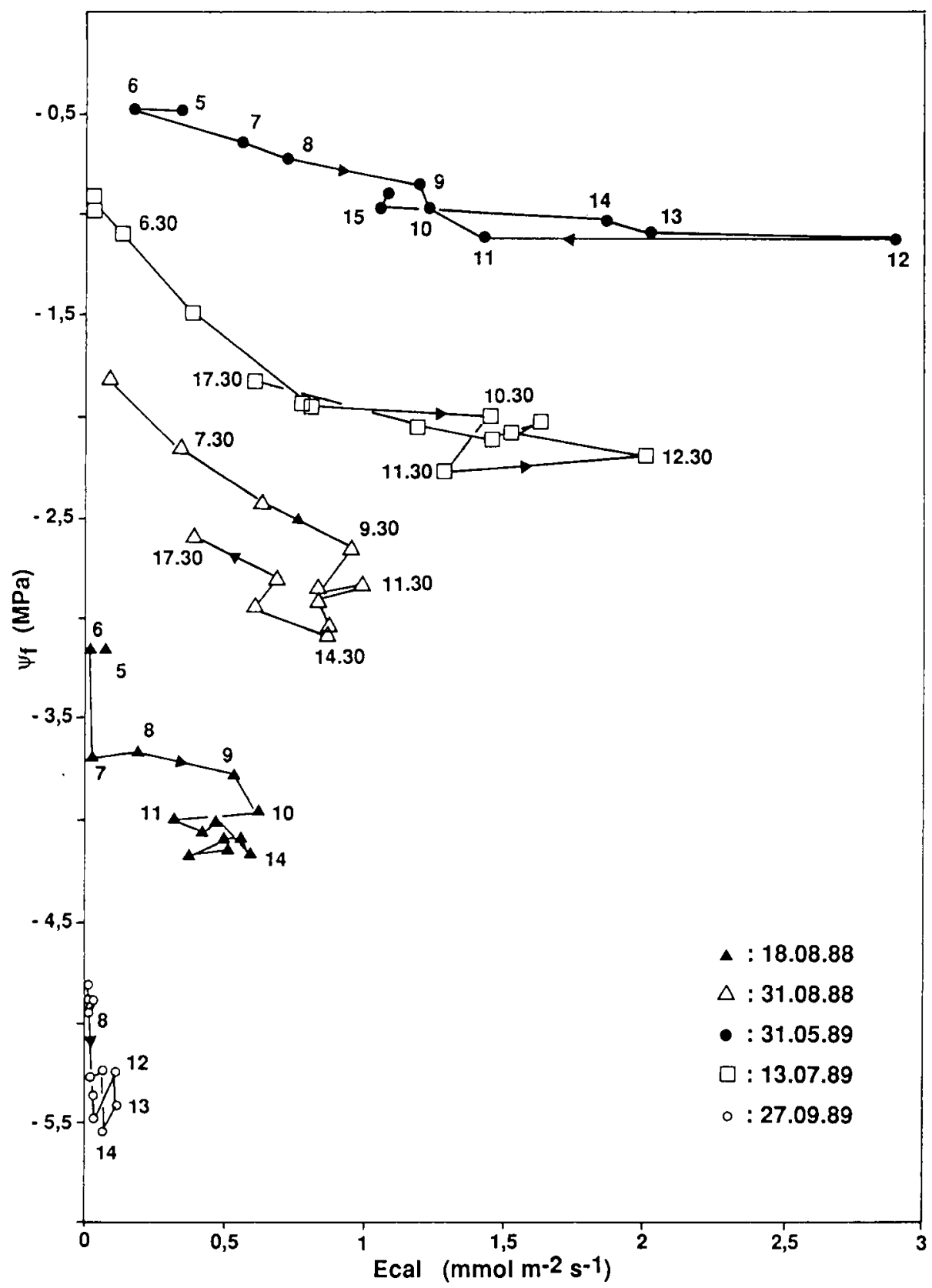

Fig 4. Exemples de relations journalières entre le potentiel hydrique foliaire $\psi_{f}$ et la transpiration $E_{c a l}$. Chaque point représente la moyenne de 5 mesures. Les courbes se lisent dans le sens chronologique indiqué par les flèches (un point par h). L'heure est indiquée à côté de chaque point. 
L'augmentation de la transpiration au cours de la journée, même si elle est minime (ex: le 27 septembre 1989), est toujours accompagnée d'une chute de potentiel. L'écart $\Delta_{\psi}$ entre le potentiel de base et le potentiel minimal ne varie pas significativement d'une journée à l'autre (tableau I) alors que la transpiration décroît de façon évidente quand la sécheresse s'accentue.

En conditions de sécheresse modérée $\left(\psi_{b}>-1,5 \mathrm{MPa}\right)$ le potentiel minimal coïncide presque exactement avec la transpiration maximale et, à transpiration égale, le potentiel est sensiblement le même avant $e$ : après ce point extrême : la relation entre $\psi_{f}$ et $E_{c a l}$ ne comporte pas d'hystérésis. Quand les conditions sont plus sévères $\left(\psi_{b}<-1,5 \mathrm{MPa}\right)$ le potentiel minimal peut être postérieur à la transpiration maximale et un effet d'hystérésis peut apparaître.

Toutes les régressions linéaires de $\psi_{f}$ sur $E_{c a l}$ sont significatives. Dans quelques cas, les points s'ajustent mieux à une relation quadratique, donc curvilinéaire, qu'à une relation linéaire; mais en aucun cas il n'y a de différence significative entre les 2 coefficients de corrélation. En conséquence c'est la pente de la relation linéaire qui est considérée comme représentant la résistance hydraulique sol-feuille (Reich et Hinckley, 1989). L'inverse de cette résistance est la conductance hydraulique solfeuille (tableau II). De façon hautement significative, la conductance hydraulique sol-feuille diminue (fig 5 ) avec le potentiel de base $\psi_{f}\left(\tau=0,943^{\star \star}\right)$.

\section{Relation entre le potentiel hydrique foliaire $\psi_{f}$ et la conductance stomatique $g_{s}$ (fig 6)}

Dans tous les cas, l'augmentation de $g_{s}$ est accompagnée d'une chute de $\psi_{f}$. Les différences concernent les événements postérieurs au maximum de $g_{s}$. En bonnes conditions d'humidité du sol (le 31 mai 1989 ainsi que le 14 juin 1989, non présenté) le $\psi_{f}$ ne décroît pas, ou à peine, au-dessous de la valeur qu'il a atteinte au moment du maximum de $g_{s}$; la réduction de la conductance freine suffisamment la transpiration pour stopper la chute de $\psi_{f}$. Au contraire, quand les conditions hydriques édaphiques sont plus sévères (ex: le 12 septembre 1988 et le 18 août 1988) le $\psi_{f}$ continue de décroître pendant que les stomates se ferment et cette décroissance supplémentaire peut atteindre $-0,7 \mathrm{MPa}$; la réduction de conductance ne freine pas suffisamment la transpiration pour stopper la chute de $\psi_{f}$. En conditions de sécheresse extrême (le 27 septembre 1989) le blocage de $\psi_{f}$ réapparaît.

Le caractère le plus remarquable de la relation de $\psi_{f}$ à $g_{s}$ (fig 6 ) est que la même conductance stomatique peut être réalisée pour des potentiels extrêmement différents, suivant les conditions hydriques du sol. Ceci suggère qu'un ajustement osmotique se produit au cours des périodes de sécheresse.

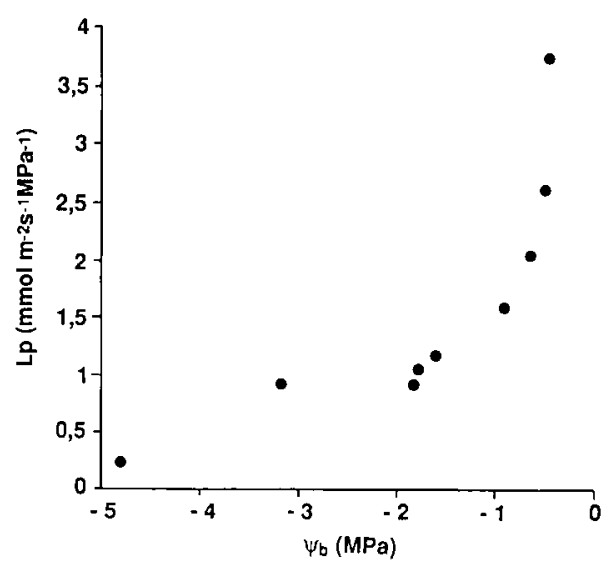

Fig 5. Relation entre la conductance hydraulique sol-feuille $L_{p}$ et le potentiel de base $\psi_{b}$. 


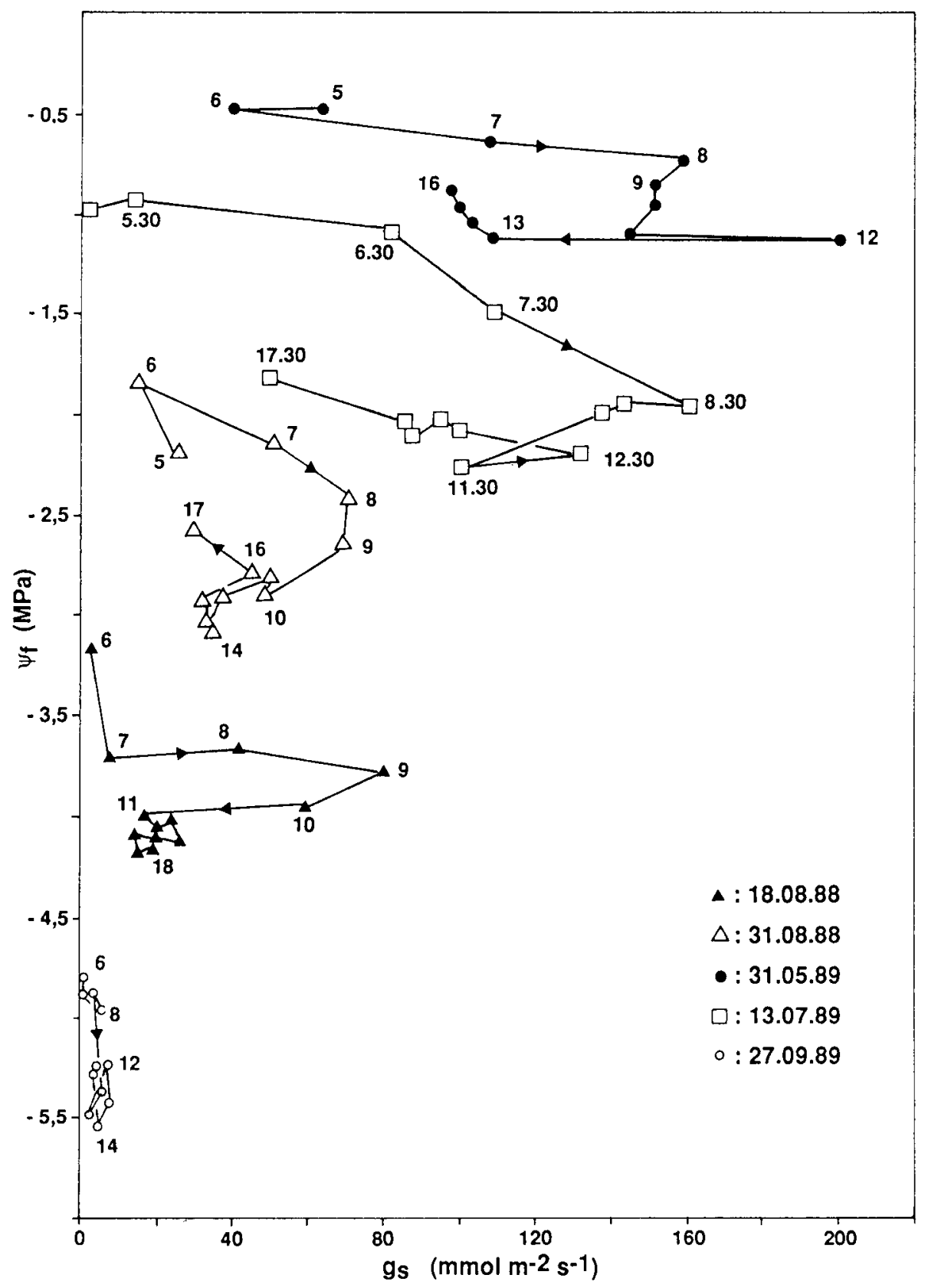

Fig 6. Exemples de relations journalières entre le potentiel hydrique foliaire $\psi_{f}$ et la conductance stomatique $g_{s}$. Chaque point représente la moyenne de 5 mesures. Les courbes se lisent dans le sens chronologique indiqué par les flèches (un point par h). L'heure est indiquée à côté de chaque point. 


\section{Indices de l'ajustement osmotique}

Cette recherche, exécutée au cours de l'été 1990, a consisté à comparer le potentiel osmotique à l'état saturé $\psi_{s o}$ et en limite de turgescence $\psi_{\text {sl }}$, pour des folioles récoltées sur le même arbre soumis à 2 conditions hydriques très différentes (tableau III). Les potentiels de base sont inconnus mais voisins des potentiels du sol à $30 \mathrm{~cm}$ de profondeur. La baisse de $\psi_{s o}$ et de $\psi_{s /}$ accompagnant l'assèchement du sol du 28 juin au 7 septembre indique l'existence de l'ajustement osmotique.

\section{DISCUSSION}

Le premier objectif de ce travail était d'obtenir des données quantitatives permettant de comparer le comportement hydrique du frêne en formation montagnarde mésoxérophile avec celui du frêne de forêt alluviale (Besnard et Carlier, 1990). Les valeurs extrêmes des grandeurs pertinentes, mesurées en l'absence de dommages visibles immédiats, sont portées au tableau IV.

Le frêne de forêt alluviale enfonce ses racines jusqu'à $1,25 \mathrm{~m}$ de profondeur.

Tableau III. Potentiels osmotiques en début et en fin de saison sèche.

\begin{tabular}{llll}
\hline Dates & $\begin{array}{l}\psi_{\text {sol }} \\
\dot{a}-30 \mathrm{~cm} \\
(\mathrm{MPa})\end{array}$ & $\begin{array}{l}\psi_{\text {so }} \\
\text { des feuilles saturées } \\
(\mathrm{MPa})\end{array}$ & $\begin{array}{l}\psi_{\text {st }} \\
\text { en limite de turgescence } \\
(\mathrm{MPa})\end{array}$ \\
\hline $\begin{array}{l}28 \text { juin } 1990 \\
07 \text { sept } 1990\end{array}$ & $-0,5$ & $-1,67 \pm 0,081$ & $-2,0$ \\
\hline
\end{tabular}

${ }^{1}$ Moyenne \pm écart type.

Tableau IV. Valeurs extrêmes mesurées chez le frêne montagnard ${ }^{1}$ el chez le frêne de forêt alluviale ${ }^{2}$.

\begin{tabular}{lcc} 
Variables & Frêne montagnard & Frêne alluvial \\
\hline$\psi_{b}(\mathrm{MPa})$ & $-0,48-4,80$ & $-0,08-0,20$ \\
$\psi_{\text {min }}(\mathrm{MPa})$ & $-1,13-5,54$ & $-0,96-1,94$ \\
$\Delta_{\psi}=\psi_{b}-\psi_{\text {min }}(\mathrm{MPa})$ & $0,65-1,34$ & $0,85-1,79$ \\
$g_{\text {smax }}\left(\mathrm{mmol} \cdot \mathrm{m}^{-2} \cdot \mathrm{s}^{-1}\right)$ & $6,6-200$ & $476-807$ \\
$E_{\text {cal }}$ maximum $\left(\mathrm{mmol}^{-2} \mathrm{~m}^{-2} \cdot \mathrm{s}^{-1}\right)$ & $0,13-2,9$ & $5,6-12,4$ \\
Conductance hydraulique & & 10,8 \\
sol-feuille $\left(\mathrm{mmol} \cdot \mathrm{m}^{-2} \cdot \mathrm{s}^{-1} \mathrm{MPa}^{-1}\right)$ & $0,9-3,7$ & \\
\hline
\end{tabular}

\footnotetext{
${ }^{1}$ Présent travail; ${ }^{2}$ Besnard et Carlier, 1990.
} 
Dans la zone des racines, le potentiel hydrique du sol ne tombe jamais au-dessous de $-0,1 \mathrm{MPa}$, grâce aux remontées capillaires s'effectuant à partir de la nappe phréatique. Le frêne montagnard installé sur éboulis de blocs enfonce ses racines jusqu'à $0,4 \mathrm{~m}$ et prélève l'eau du sol au voisinage de $0,3 \mathrm{~m}$ où le potentiel peut, en cas de sécheresse prolongée, s'ab aisser à -5 MPa. En conséquence, le frêne de forêt alluviale n'est jamais soumis à la sécheresse édaphique alors que le frêne montagnard l'a été, dans le biotope choisi ici, 3 étés de suite $(1988,1989,1990)$.

Le frêne de plaine est réputé gros consommateur d'eau (Ladefoged, 1963; Aussenac et Lévy, 1983), réputation méritée également par le frêne de forêt alluviale (tableau IV). Le frêne montagnard est beaucoup plus sobre, même en bonnes conditions d'humidité du sol, et restreint notablement sa transpiration en période de sécheresse. Cette différence repose sur celle des conductances stomatiques maximales (tableau IV) et sur la cinétique diurne de l'ouverture stomatique. Le frêne de forêt alluviale garde ses stomates grand ouverts une bonne partie de la journée, sauf en cas de diminution notable de l'ensoleillement; le frêne montagnard les ferme partiellement, au plus tard à midi et habituellement beaucoup plus tôt (tableau I).

Les jours chauds et secs où sa transpiration est intense, le frêne de forêt alluviale subit une chute abrupte du potentiel hydrique foliaire sans qu'intervienne aucune régulation stomatique. Cependant, grâce aux bonnes conditions édaphiques et à une conductance hydraulique sol-feuille remarquablement élevée, le $\psi_{f}$ minimal ne tombe pas au-dessous de $-2 \mathrm{MPa}$. Le frêne montagnard fait l'inverse. Sous l'effet dominant de la sécheresse du sol, le $\psi_{f}$ de ses feuilles peut atteindre des valeurs très basses, plus proches de celles d'arbres de régions arides ou désertiques (Roberts et al, 1981; Nilsen et al, 1984) que de celles de feuillus de régions tempérées (Hinckley et al, 1978). En revanche, la chute diurne de $\psi_{f}$ est plus modérée que celle du frêne de forêt alluviale, grâce à la fermeture partielle des stomates; toutefois cette régulation n'a qu'une efficacité limitée puisqu'à compter de son déclenchement, il peut encore se produire une chute de potentiel atteignant $-0,7 \mathrm{MPa}$.

Au vu de ces différences de comportement entre frêne de forêt alluviale et frêne de montagne, une étude génétique comparée serait justifiée.

Un second domaine de discussion concerne la signification adaptative des propriétés mises en évidence.

Les stomates s'ouvrent d'autant moins que la sécheresse est plus prononcée comme le montre la corrélation très significative entre la conductance maximale et le potentiel de base, déjà établie pour d'autres arbres (Reich et Hinckley, 1989). En outre, quand le $\psi_{b}$ s'abaisse, l'efficacité de l'éclairement sur l'ouverture stomatique diminue (tableau III). Davies et Kozlowski (1975) ont montré que la réponse des stomates aux changements d'éclairement est influencée négativement par la détresse hydrique chez plusieurs arbres, à des degrés divers selon l'espèce (Fraxinus americana est relativement peu sensible).

Ces éléments du comportement du frêne de montagne contribuent à limiter la transpiration mais ne limitent que très imparfaitement la diminution de $\psi_{f}$. Selon Aussenac et Granier (1978) la chute diurne de potentiel foliaire $\Delta \psi$ est limitée à $0,4 \mathrm{MPa}$ quand la transpiration est exclusivement cuticulaire; des arbres méditerranéens ont même un $\Delta \psi$ nul en période estivale (Morrow et Mooney, 1974). Chez notre frêne montagnard, cette situation n'a jamais été observée: même en cas de sé- 
cheresse extrême, une ouverture stomatique significative se produit et $\Delta \psi$ atteint $0,74 \mathrm{MPa}$.

Une autre propriété peu favorable à la stabilisation du $\psi_{f}$ réside dans la conductance hydraulique sol-feuille (tableau IV). Non seulement, celle-ci, dans les meilleur 3 conditions, est 3 fois plus faible chez le frêne montagnard que chez le frêne de forêt alluviale, ce qui, à transpiration égale, favorise la chute du $\psi_{t}$, mais encore on observe une diminution progressive de cette conductance quand la sécheresse s'accentue. Selon Reich et Hinckley (1989), cette diminution de la conductance hydraulique peut être due à l'augmentation de la résistance hydraulique du sol et à des mécanismes propres à l'arbres et donc susceptibles de variations selon l'espèce et le milieu, tels que la disparition des fines racines et la cavitation. Ce dernier mécanisme serait prépondérant chez la vigne (Schultz et Matthews, 1988).

Ainsi, parmi les stratégies adaptatives définies par Ludlow (1989), le frêne montagnard ne pratique pas celle de l'évitement puisqu'il ne possède pas de dispositif adéquat pour limiter la chute de son potentiel hydrique foliaire. II survivrait donc aux épisodes de sécheresse estivale par la stratégiu de tolérance. On peut observer que notre frêne a supporté sans dommage, le 27 septembre 1989, des potentiels foliaires allant de $-4,8$ à $-5,5 \mathrm{MPa}$ pendant que d'autres individus du même biotope subissaient des dommages (constatés plusieurs jours plus tard). Le potentiel critique défini par Levitt (1963), correspondant à un seuil létal, a donc été approché.

L'un des éléments de la stratégie de tolérance est l'ajustement stomatique, "processus par lequel les réponses stomatiques sont modifiées pour être appropriées à la nouvelle situation de détresse hydrique" (Ludlow, 1980). Le frêne de montagne pratique l'ajustement stomatique puisque la relation entre $g_{s}$ et $\psi_{f}$ se modifie avec l'avancement de la sécheresse (fig 6). Un mécanisme possible de l'ajustement stomatique est l'ajustement osmotique (Ludlow, 1980; Hinkley et al, 1980). L'ajustement osmotique favorise le maintien de la turgescence. II est mesuré par l'abaissement du potentiel osmotique dans des situations précises, par exemple à pleine turgescence ou à la limite de perte de turgescence, le second critère étant plus sensible (Turner et Jones, 1980). C'est bien ce qui se produit chez le frêne de montagne (tableau III). L'ajustement osmotique a lieu chez l'abricotier (Loveys et al, 1987) et aussi, en même temps que l'ajustement stomatique, chez Cornus sanguinea, alors que l'un et l'autre manquent chez d'autres arbres, dont l'olivier (Hinckley et al, 1980).

Un dernier point à discuter se rapporte au déterminisme des mouvements stomatiques, mais il ne faut pas perdre de vue qu'un travail comme celui-ci, effectué en conditions naturelles incontrôlables, n'est pas particulièrement approprié à la mise en évidence des facteurs responsables.

L'éclairement intervient de façon prépondérante en début et en fin de journée. L'efficacité lumineuse, qui mesure la sensibilité des stomates à l'augmentation matinale de l'éclairement, est en corrélation significative avec le potentiel de base (tableau II) comme l'est aussi, de façon très significative, la conductance maximale. Ces faits peuvent s'interpréter, à la suite de Gollan et al (1985), comme indiquant une dépendance du fonctionnement stomatique par rapport aux conditions hydriques régnant dans le sol au voisinage des racines. Le signal transmis des racines aux feuilles pourrait être constitué par la variation de concentration de l'acide abscissique dans la sève brute (Wartinger 
et al, 1990). Cependant Reich et Hinckley (1989) suggèrent que le signal pourrait être constitué par la valeur de la conductance hydraulique sol-feuille. Effectivement, chez le frêne montagnard, il existe des corrélations très significatives, d'une part entre la conductance stomatique maximale et la conductance hydraulique sol-feuille $\left(\tau=0,833^{* \star}\right)$, d'autre part entre la conductance hydraulique sol-feuille et le potentiel de base $\left(\tau=0,943^{\star \star}\right.$; tableau II, fig 5).

Par ailleurs, la cinétique diurne de la conductance stomatique est dominée par l'existence d'un "décrochement» dans la relation entre conductance et éclairement (phase c, fig 3). Quel facteur en est responsable? Une littérature abondante se rapporte à la détermination du potentiel foliaire "seuil" au-delà duquel la fermeture des stomates s'amorce (Hinckley et al, 1978) ce qui implique que le potentiel foliaire agit instantanément sur les stomates (Ludlow, 1980), hypothèse récusée par Gollan et al (1985) et par Wartinger et al (1990). Dans le cas du frêne montagnard, il n'est pas possible d'associer le "décrochement" à une valeur fixe de $\psi_{f}$. Les recherches relatives à l'impact de I'ABA, apporté aux feuilles par la sève brute, sur la cinétique diurne des stomates n'ont pas permis de conclure positivement (Loveys et Düring, 1984; Zhang et Davies, 1989). Enfin, l'influence du VPD atmosphérique sur les stomates a été démontrée dans de nombreux cas (Levy, 1980; Körner et Cochrane, 1985) mais nos données ne font pas apparaître d'effet de ce genre chez le frêne de montagne, pas plus que chez le frêne de forêt alluviale (Besnard et Carlier, 1990).

\section{CONCLUSION}

Le frêne montagnard mésoxérophile est moindre gaspilleur d'eau que le frêne de forêt alluviale, grâce à une ouverture stomatique moins grande et moins durable, et sensible à l'assèchement du sol. Dans une certaine mesure, il ménage la réserve d'eau limitée présente dans le sol peu abondant des éboulis qu'il occupe. Toutefois, son maintien dans de tels biotopes, au cours des épisodes de sécheresse estivale fréquents dans les Alpes intermédiaires, ne repose pas tant sur l'évitement que sur des propriétés de tolérance dont les limites restent à déterminer.

\section{REMERCIEMENTS}

Nous remercions $M$ le Maire d'Huez-en-Oisans qui a facilité la recherche du site, le Dr B Doche qui nous a conseillés dans le choix de celui-ci, et Mme E Sarret qui a autorisé les travaux expérimentaux sur la parcelle dont elle est propriétaire.

\section{RÉFÉRENCES}

Aussenac G, Granier A (1978) Quelques résultats de cinétique journalière du potentiel de sève chez les arbres forestiers. Ann Sci For 35, $19-32$

Aussenac G, Lévy G (1983) Influence du dessèchement du sol sur le comportement hydrique et la croissance du chêne pédonculé (Quercus pedunculata Ehrl) et du frêne (Fraxinus excelsior L) cultivés en cases de végétation. Ann Sci For 40, 251-264

Besnard G, Carlier G (1990) Potentiel hydrique et conductance stomatique des feuilles de frêne (Fraxinus excelsior $\mathrm{L}$ ) dans une forêt alluviale du Haut-Rhône français. Ann Sci For 47, 353-365

Braun HJ (1977) Growth and water economy of the trees Acer pseudoplatanus $\mathrm{L}$ and Fraxinus excelsior L. Z Pflanzenphysiol 84, 459462

Bruckler L (1984) Utilisation des micropsychromètres pour la mesure du potentiel hydrique du sol en laboratoire et in situ. Agronomie 4, 171-182 
Davies WJ, Kozlowski TT (1975) Stomatal responses to changes in light intensity as influenced by plant water stress. For Sci 21 , 129-133

Devauchelle R, Lévy G (1977) Propriétés stationnelles et croissance du frêne dans l'Est de la France, étude de certaines caractéristiques de cette essence. Ann Sci For 34, 231244

Gollan T, Turner NC, Schulze ED (1985) The response of stomata and leaf gas exchange to vapor pressure deficits and soil water content. 3. In the sclerophyllous woody species Nerium oleander. CEcologia 65, 356-362

Hinckley TM, Lassoie JP, Running SW (1978) Temporal and spatial variations in the water status of forest trees. For Sci 24 , Monograph $20,79 p$

Hinckley TM, Duhme F, Hinckley AR, Richter $H$ (1980) Water relations of drought hardy shrubs: osmotic potential and stomatal reactivity. Plant Cell Environ 3, 131-140

Körner C, Cochrane PM (1985) Stomatal responses and water relations of Eucalyptus pauciflora along an elevational gradient. CEcologia 6, 443-455

Ladefoged K (1963) Transpiration of forest trees in closed stands. Physiol Plant 16, 378-414

Le Goff N, Lévy G (1984) Productivité du frêne (Fraxinus excelsior L) en région NordPicardie. B. Étude des relations entre la productivité et les conditions de milieu. Ann Sci For 41, 135-170

Levitt J (1963) The measurement of drought resistance. In: Methodology of plant ecophysiology (Eckardt FE, ed) Proc Montpellier Symp, Unesco 407-412

Levy $Y(1980)$ Effect of evaporative demand on water relations of Citrus limonum. Ann Bot 46, 695-700

Loveys BR, Düring $H$ (1984) Diurnal changes in water relations and abscissic acid in fieldgrown Vitis vinifera cultivars. New Phytol 97 , 37-47

Loveys BR, Robinson SP, Downton WJS (1987) Seasonal and diurnal changes in abscissic acid and water relations of apricot leaves (Prunus armeniaca L). New Phytol 107, $15-27$
Ludlow M (1980) Significance of stomatal response to water stress. In: Adaptation of plants to water and high temperature stress (Turner NC, Kramer PJ, eds) New York 123138

Ludlow M (1989) Strategies of response to water stress. In: Structural and functional responses to environmental stresses. (Kreeb $\mathrm{KH}$, Richter $\mathrm{H}$ et Hinckley TM, eds) SPB Acad Publ bv, La Haye, 269-281

Morrow PA, Mooney HA (1974) Drought adaptation in two California evergreen sclerophylls. CEcologia 15, 205-222

Nilsen ET, Sharf MR, Rundel PW (1984) Comparative water relations of phreatophytes in the Sonoran desert of California. Ecology 65, 767-778

Ozenda $\mathrm{P}$ (1985) La végétation de la chaîne alpine dans l'espace montagnard européen. Masson, Paris, 25

Pautou G (1970) Écologie des formations alluviales de la Basse Isère. Application à l'étude d'une nappe phréatique et de ses risques de pollution. Documents pour la carte de la végétation des Alpes VIII, 73-114

Pautou G, Cadel G, Girel R (1992) Le bassin de Bourg d'Oisans, un carrefour phytogéographique des Alpes françaises. Rev Ecol Alpine 1, 23-43

Reich PB, Hinckley TM (1989) Influence of predawn water potential and soil-to-leaf hydraulic conductance on maximum daily leaf diffusive conductance in two oak species. Funct Ecol 3, 719-726

Roberts SW, Miller PC, Valamanesh A (1981) Comparative field water relations of four $\mathrm{CO}^{-}$ occurring chaparral species. CEcologia 48, 360-363

Scholander PM, Hammel HT, Bradstreet ED, Hemmingsen EA (1965) Sap pressure in vascular plants. Science 148, 339-346

Schultz HR, Matthews MA (1988) Resistance to water transport in shoots of Vitis vinifera $L$. Relation to growth at low water potential. Plant Physiol 88, 718-724

Sokal RR, Rohlf FJ (1981) Biometry. The principles and practice of statistics in biological research. Freeman and Co, $859 \mathrm{p}$ 
Turner NC, Jones MM (1980) Turgor maintenance by osmotic adjustment: a review and evaluation. In: Adaptation of plants to water and high temperature stress (Turner NC, Kramer PJ, eds) New York, 87-104

Turner NC, Kramer PJ (eds) (1980) Adaptation of plants to water and high temperature stress. New York, 1-5

Wartinger A, Heilmeier $\mathrm{H}$, Hartung $\mathrm{W}$, Schulze ED (1990) Daily and seasonal courses of leaf conductance in the xylem sap of almond trees (Prunus dulcis [Miller] DA Webb) under desert conditions. New Phytol 116, 581-587

Zhang J, Davies WJ (1989) Sequential response of whole plants water relations to prolonged soil drying and the involvment of xylem sap $A B A$ in the regulation of stomatal behaviour on sunflower plants. New Phytol $113,167-174$

\section{ANNEXES}

$E_{\text {cal }}$ : intensité de la transpiration $\left(\mathrm{mmol}\left[\mathrm{H}_{2} \mathrm{O}\right] \mathrm{m}^{-2}\right.$ . $\left.\mathrm{s}^{-1}\right)$

$g_{s}$ : conductance stomatique pour la vapeur d'eau $\left(\mathrm{mmol}\left[\mathrm{H}_{2} \mathrm{O} \mathrm{m}^{-2} \cdot \mathrm{s}^{-1}\right)\right.$

$g_{\text {smax }}:$ conductance stomatique maximale

$Q$ : éclairement ou “irradiance" ( $\mu$ mol[photons] $\mathrm{m}^{-2} \cdot \mathrm{s}^{-1}$ )

RWC : teneur en eau relative (\%)

VPD : déficit de pression de vapeur d'eau atmosphérique ( $\mathrm{hPa}$ )

$\psi_{b}$ : potentiel hydrique foliaire de base (MPa). $\psi_{f}$ : potentiel hydrique foliaire $(\mathrm{MPa}) . \psi_{\min }: \mathrm{po}-$ tentiel hydrique foliaire minimum (MPa). $\psi_{\text {sol }}$ : potentiel hydrique du sol $(\mathrm{MPa}) . \psi_{s}$ : potentiel osmotique (MPa).

$\Delta \psi=\psi_{b}-\psi_{\text {fmin }}$ 